\title{
Effects of composition, temperature, and magnetism on phonons in bcc Fe-V alloys
}

\author{
M. S. Lucas, ${ }^{1,2}$ J. A. Muñoz, ${ }^{3}$ O. Delaire, ${ }^{1}$ N. D. Markovskiy, ${ }^{3}$ M. B. Stone, ${ }^{1}$ D. L. Abernathy,,${ }^{1}$ I. Halevy, ${ }^{3}$ L. Mauger, ${ }^{3}$ \\ J. B. Keith, ${ }^{3}$ M. L. Winterrose,${ }^{3}$ Yuming Xiao, ${ }^{4}$ M. Lerche, ${ }^{5}$ and B. Fultz ${ }^{3}$ \\ ${ }^{1}$ Oak Ridge National Laboratory, 1, Bethel Valley Road, Oak Ridge, Tennessee 37831, USA \\ ${ }^{2}$ Air Force Research Laboratory, Wright-Patterson AFB, Ohio 45433, USA \\ ${ }^{3}$ California Institute of Technology, W. M. Keck Laboratory, 138-78, Pasadena, California 91125, USA \\ ${ }^{4}$ HPCAT, Geophysical Laboratory, Carnegie Institute of Washington, Argonne, Illinois 60439, USA \\ ${ }^{5}$ High Pressure Synergetic Consortium, Carnegie Institute of Washington, Argonne, Illinois 60439, USA
}

(Received 20 August 2010; published 22 October 2010)

\begin{abstract}
The phonon densities of states of body-centered-cubic Fe-V alloys across the full composition range were studied by inelastic neutron scattering, nuclear resonant inelastic x-ray scattering, and ab initio calculations. The average phonon energy followed the inverse of the electronic heat capacity and the inverse of the electronic density of states at the Fermi level, showing how the interatomic forces depend on electronic screening. These quantities, including phonon energy, changed rapidly near the composition of the paramagneticferromagnetic transition. For Fe- and V-rich alloys, the thermal phonon softening deviated from quasiharmonic behavior but better agreement was found for intermediate compositions. The Fe partial phonon density of states has a distinctly different shape than $\mathrm{V}$ for alloys with less than 50 at. \% Fe.
\end{abstract}

DOI: $10.1103 /$ PhysRevB.82.144306

PACS number(s): 63.20.dd, 63.20.kd, 71.20.Be, 75.50.Bb

\section{INTRODUCTION}

Phonon thermodynamics underlies the vibrational entropy of materials, which contributes substantially to alloy phase stability and phase transitions. ${ }^{1}$ Understanding how composition, structure, temperature, and pressure alter the phonon frequencies has become a rich topic for materials research. ${ }^{2-7}$ Unfortunately, simple rules derived from atomic mass, size, electronegativity, electron-to-atom ratio, and bond lengths are at best semiquantitative. Quantitative predictions must address the details of the electronic structure and how it depends on nuclear positions. This includes the effects of magnetism, which alter substantially the electron density at the Fermi level.

The body-centered-cubic (bcc) phase in the Fe-V alloy system spans across the full composition range. ${ }^{8,9}$ Systematic changes in magnetic properties with composition make Fe-V alloys interesting for a study of the effects of magnetism on phonon thermodynamics. The magnetic moment decreases linearly from the value of bulk $\mathrm{Fe}$ as the $\mathrm{V}$ concentration increases until it disappears at about 70 at. $\% \mathrm{~V}$ at low temperature. ${ }^{10-12}$ The Curie temperature is $300 \mathrm{~K}$ at approximately 58 at. $\%$ V. ${ }^{13,14}$ First-principles calculations by several methods have successfully reproduced the trends in the magnetism $^{15-18}$ and predict a strong hybridization of the atomic orbitals, charge transfer from the $\mathrm{V}$ to the Fe atoms, and the development of an antiparallel moment at the $\mathrm{V}$ atoms. The latter was confirmed by the neutron diffuse scattering measurements of Mirebeau and Parette. ${ }^{19}$ The total energy calculations as a function of volume for pure $\mathrm{Fe}$ performed by Moruzzi and Marcus, ${ }^{16}$ predict the spinpolarized phase to be stable over the nonspin-polarized phase. These calculations showed that the electronic energies vary more with volume in the nonspin-polarized phase, resulting in a higher bulk modulus than the ferromagnetic phase.

When an atom is displaced from its equilibrium position, the conduction electrons are free to screen the corresponding charge disturbance. The better the screening, the lower the electronic energy and interatomic forces. It is generally expected that a metal with a large electronic density of states (eDOS) at the Fermi level, $n\left(E_{\mathrm{F}}\right)$, should have phonons of lower average energy than a material with small $n\left(E_{\mathrm{F}}\right) \cdot{ }^{20}$ The full picture requires details about how the different electron states at the Fermi level respond to atom displacements, and this is complicated for transition metal alloys. Nevertheless, when large, sharp features are present in $n(E)$ near $E_{\mathrm{F}}$, changes in composition and temperature can alter significantly the phonon dispersions and the phonon density of states (pDOS). For pure V, the adiabatic electron-phonon interaction (EPI) broadens the eDOS, causing a decrease in $n\left(E_{\mathrm{F}}\right)$ with increasing temperature. ${ }^{21} \mathrm{~A}$ consequence is a reduction in electronic screening with temperature so the phonon DOS remains anomalously stiff at elevated temperatures. These effects on the phonons are of interest for their own sake but they also have thermodynamically significant effects on the vibrational entropy.

Both $\mathrm{Fe}$ and $\mathrm{V}$ have similar molecular weights, so mass effects from compositional changes are expected to shift phonon frequencies $(\Delta \omega / \omega)$ by less than $5 \%$. Larger effects are expected from changes in volume with temperature and perhaps composition. The effects of volume on the phonons are first accounted for with the quasiharmonic $(\mathrm{QH})$ model. In this model the phonons are assumed harmonic but increases in volume owing to thermal expansion cause decreases in phonon energies (in proportion to a Grüneisen parameter). We might expect similar effects from the changes of volume with alloy composition, although this is less direct. The specific volume of $\mathrm{Fe}_{1-x} \mathrm{~V}_{x}$ alloys increases monotonically with $x$, where vanadium is $\sim 18 \%$ larger than Fe.

In this study, we explore the effects of alloying and temperature on the phonon density of states of bcc $\mathrm{Fe}_{1-x} \mathrm{~V}_{x}$ across the entire composition range $(0<x<1)$. In what follows we explain the inelastic scattering techniques and their results, present predictions from quasiharmonic theory, and 
describe results from $a b$ initio calculations of electronic structure and phonon dynamics. There are some successes of quasiharmonic theory for thermal trends at intermediate compositions. The compositional trends, however, deviate substantially from QH theory at the ferromagnetic transition. This effect seems dominated by changes in the eDOS at the Fermi level.

\section{EXPERIMENTS AND RESULTS}

For the neutron-scattering measurements, alloys of $\mathrm{Fe}_{1-x} \mathrm{~V}_{x}$ for $x=\{1,0.94,0.75,0.50,0.25,0\}$ were prepared by arc melting under an argon atmosphere using shots of $99.99 \% \mathrm{Fe}$ and $99.8 \% \mathrm{~V}$. The ingots were cold rolled to thicknesses corresponding to a neutron-scattering probability of $10 \%(1-3 \mathrm{~mm})$. The rolled strips were annealed in evacuated quartz ampoules to produce bcc solid solutions. X-ray diffraction patterns were acquired at room temperature for all samples and confirmed the bcc structure. There was no measurable evidence of the sigma phase or oxidation. For the inelastic $\mathrm{x}$-ray measurements, alloys of $\mathrm{Fe}_{1-x} \mathrm{~V}_{x}$ for $x$ $=\{0.99,0.95,0.90,0.80,0.70,0.50,0.25,0\}$ were prepared similarly to the INS samples but $96.06 \%$ enriched ${ }^{57} \mathrm{Fe}$ was substituted for natural Fe. After melting, the small ingots were cold rolled to a thickness of 20-50 $\mu \mathrm{m}$. Electron microprobe measurements confirmed the compositions to be accurate to 0.5 at. $\%$.

Inelastic neutron-scattering (INS) spectra were measured with the wide angular-range chopper spectrometer at the Spallation Neutron Source (SNS) of the Oak Ridge National Laboratory. Samples were surrounded by thin-wall aluminum pans of area $5 \mathrm{~cm} \times 7 \mathrm{~cm}$, and mounted in the $\mathrm{Cu}$ frame of a custom-built low-background electrical resistance furnace, which was kept under high vacuum during all measurements. The flat sample packages were tilted at a $45^{\circ}$ angle off the direction of the incident beam to minimize self shielding. All samples (with the exception of $\mathrm{Fe}_{0.50} \mathrm{~V}_{0.50}$, which develops chemical order) were measured at 300 and $773 \mathrm{~K}$. The samples were allowed to cool under vacuum and remeasured at $300 \mathrm{~K}$. Neutron diffraction, acquired from the elastic scattering, indicated no change in the samples after the heating to $773 \mathrm{~K}$. No oxidation was observed on the samples after the measurements. The lattice parameters from neutron diffraction were consistent with those determined from $\mathrm{x}$-ray diffraction and consistent with values from the literature. ${ }^{8}$ All measurements were performed with an incident beam of monochromatic neutrons with a nominal energy of $80 \mathrm{meV}$ and chopper frequency of $600 \mathrm{~Hz}$. The energy resolution [full width at half maximum (FWHM)] was $1.7 \mathrm{meV}$ at $40 \mathrm{meV}$ neutron energy loss, increasing to 3.1 $\mathrm{meV}$ at the elastic line. The empty Al sample container was measured in identical conditions at all temperatures and was subtracted from the measured spectra of the samples.

Nuclear resonant inelastic $x$-ray scattering (NRIXS) (Refs. 22-24) was performed at beamline 16ID-D at the Advanced Photon Source (APS) of the Argonne National Laboratory. The incident photon energy was tuned to $14.413 \mathrm{keV}$, the nuclear resonance energy of ${ }^{57} \mathrm{Fe}$. Each sample was placed on Kapton tape at a grazing angle to the incident

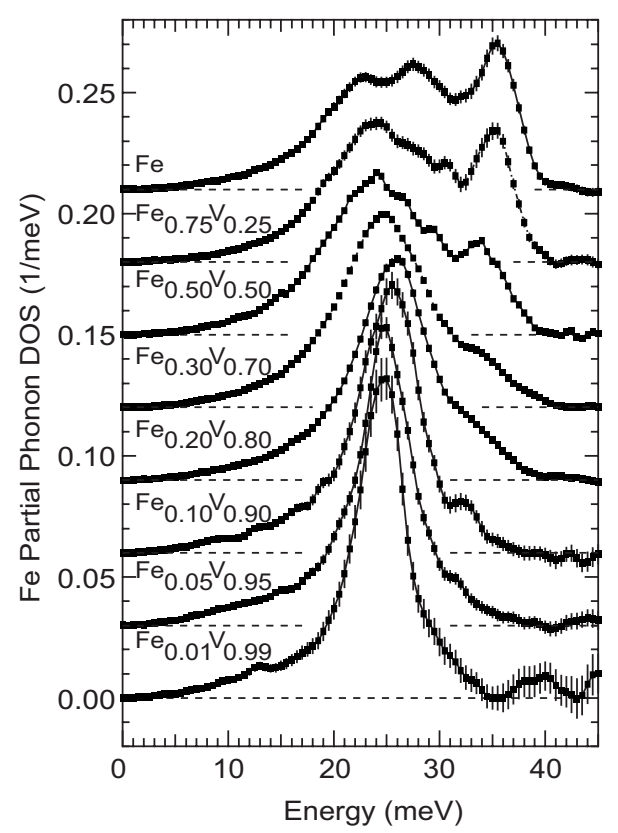

FIG. 1. ${ }^{57} \mathrm{Fe}$ pDOS curves from NRIXS spectra at $300 \mathrm{~K}$. Curves are offset by integer multiples of $0.03 \mathrm{meV}^{-1}$.

X-ray beam. The NRIXS signal was measured with two avalanche photodiode detectors (APDs) with active areas in close proximity to each other, positioned $90^{\circ}$ from the direction of the beam. Data were collected in scans of incident photon energy from -80 to $+80 \mathrm{meV}$ around the main resonant peak. All measurements were performed at room temperature. The monochromator resolution function was measured in situ using a single APD in the forward direction. The FWHM was $\sim 2.2 \mathrm{meV}$. The NRIXS data reduction was performed with the software PHOENIX. ${ }^{25}$ The results of the inelastic X-ray scattering measurements are presented in Fig. 1. As a function of composition, there is smooth change in spectral shape from the phonon DOS of pure Fe to a peak centered at $25 \mathrm{meV}$ with a FWHM of $\sim 8 \mathrm{meV}$ for dilute concentrations of $\mathrm{Fe}$ in $\mathrm{V}$.

Details about the procedures for reducing inelastic neutron-scattering data are described elsewhere. ${ }^{5,26-28}$ Intermediate INS results are presented in Fig. 2. Further reduction generates curves $g^{\text {nw }}(E)$, as shown in Fig. 3. These curves are "neutron weighted." The undistorted total phonon DOS $g(E)$ is

$$
g(E)=\sum_{d} g_{d}(E) x_{d},
$$

where $g_{d}(E)$ are the phonon partial densities of states of atom $d$ [the Fe-specific phonon partial density of states (Fe pDOS) or $\mathrm{V}$ pDOS], and $x_{d}$ is its mole fraction. The function $g(E)$ of Eq. (1) is required for calculating thermodynamic quantities, such as the phonon entropy or phonon contribution to the heat capacity. The efficiency for the scattering of neutrons by phonons is proportional to $\sigma_{\mathrm{sc}} / m$ and the neutron-weighted DOS is 


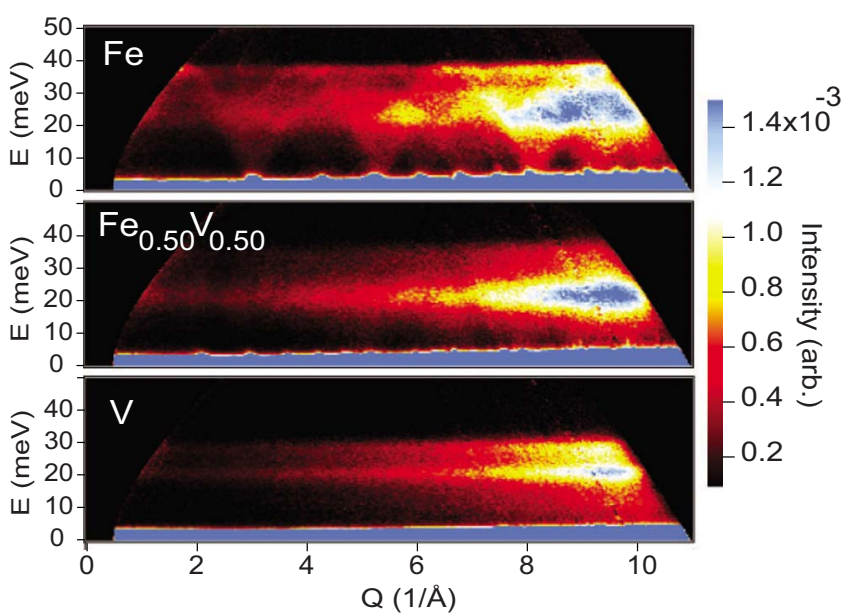

FIG. 2. (Color online) Intensity as a function of energy transfer and momentum transfer for pure $\mathrm{Fe}$ (top), $\mathrm{Fe}_{0.50} \mathrm{~V}_{0.50}$ (middle), and pure $\mathrm{V}$ (bottom) from neutron scattering at $300 \mathrm{~K}$.

$$
g^{\mathrm{nw}}(E)=A^{-1} \sum_{d} g_{d}(E) \frac{\sigma_{\mathrm{sc}, d}}{m_{d}} x_{d}
$$

where $\sigma_{\text {sc }}$ is the total scattering cross section, $m$ is the molecular weight, and $A$ is a normalization factor. For Fe and V the total scattering cross sections are 11.62 barns and 5.1 barns, respectively. The phonon modes with large motions of $\mathrm{Fe}$ atoms are therefore overemphasized in the neutronweighted DOS curves of alloys. Fortunately, the NRIXS

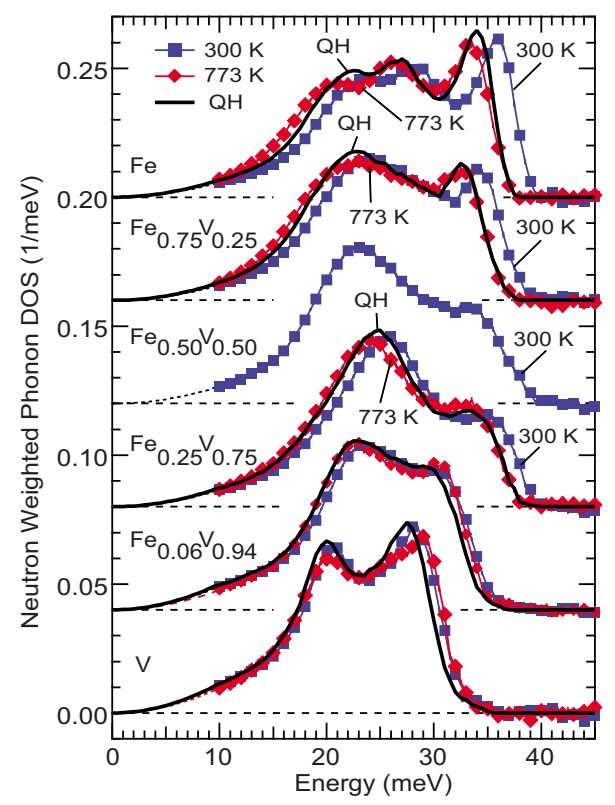

FIG. 3. (Color online) Neutron-weighted phonon density of states curves for the alloys measured as a function of temperature. Curves are offset by integer multiples of $0.04 \mathrm{meV}^{-1}$. The dashed curves up to $10 \mathrm{meV}$ indicate the Debye model fit for the lowenergy modes. Error bars are shown and are smaller than the markers. Solid lines (labeled "QH") show the $300 \mathrm{~K}$ DOS shifted in energy using the quasiharmonic model to the volume at $773 \mathrm{~K}$ (Sec. III).

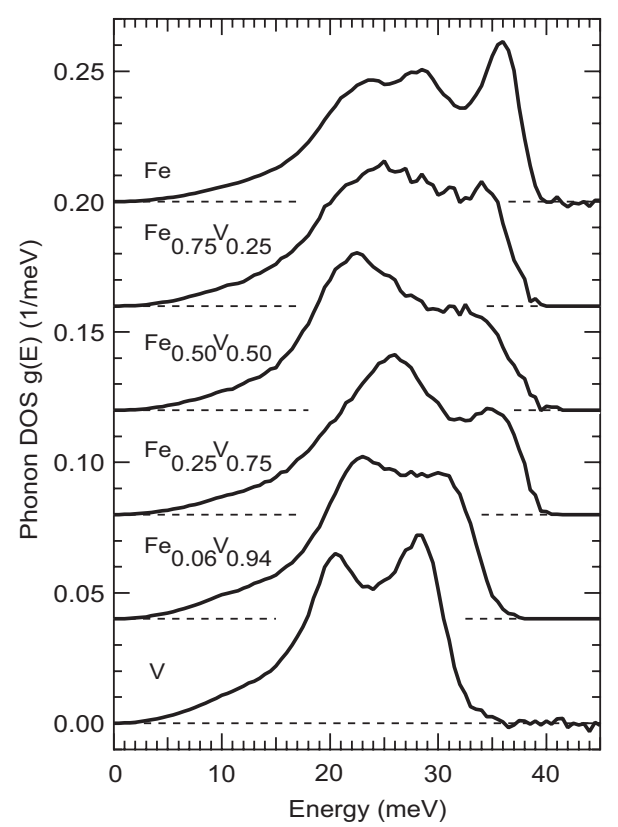

FIG. 4. Phonon DOS curves, $g(E)$, for the alloys at $300 \mathrm{~K}$, after using NRIXS results to correct for neutron weighting. Curves are offset by integer multiples of $0.04 \mathrm{meV}^{-1}$.

technique provides for a direct measurement of $g_{\mathrm{Fe}}(E)$. Subtracting the extra weight of the Fe pDOS from $g^{\mathrm{nw}}(E)$, we obtain the phonon DOS $g(E)$, corrected for neutron weighting. These $g(E)$ are presented in Fig. 4.

For pure Fe, the DOS measured by NRIXS is in good agreement with the INS results. Figure 5(a) shows the average energy of phonons, $\langle E\rangle=\int g(E) E \mathrm{~d} E$. Despite having very dissimilar shapes for the V-rich alloys, $\langle E\rangle$ is similar for the NRIXS and INS results. The V pDOS and Fe pDOS therefore have very similar average energies even though their shapes are different. A kink in $\langle E\rangle$ versus composition is observed at $x=0.25$. This is consistent with changes in a number of other properties in $\mathrm{Fe}_{1-x} \mathrm{~V}_{x}$ alloys that accompany the magnetic transition. For example, there is a change in the slope of the lattice parameter ${ }^{8,29,30}$ as a function of composition [Fig. 5(b)]. There is also a kink in the composition dependence of the electronic contribution to the lowtemperature heat capacity, ${ }^{31}$ the inverse of which is plotted against the average energy in Fig. 5(a).

\section{QUASIHARMONIC EFFECTS}

Figure 5 shows a general trend of decreasing phonon energies with increasing lattice parameter. This seems consistent with a quasiharmonic model, for which the fractional change in the phonon energy levels depends linearly on the fractional change in volume

$$
\frac{\Delta E}{E}=-\gamma \frac{\Delta V}{V},
$$

where the thermodynamic Grüneisen parameter (average over all phonon modes) is given by 


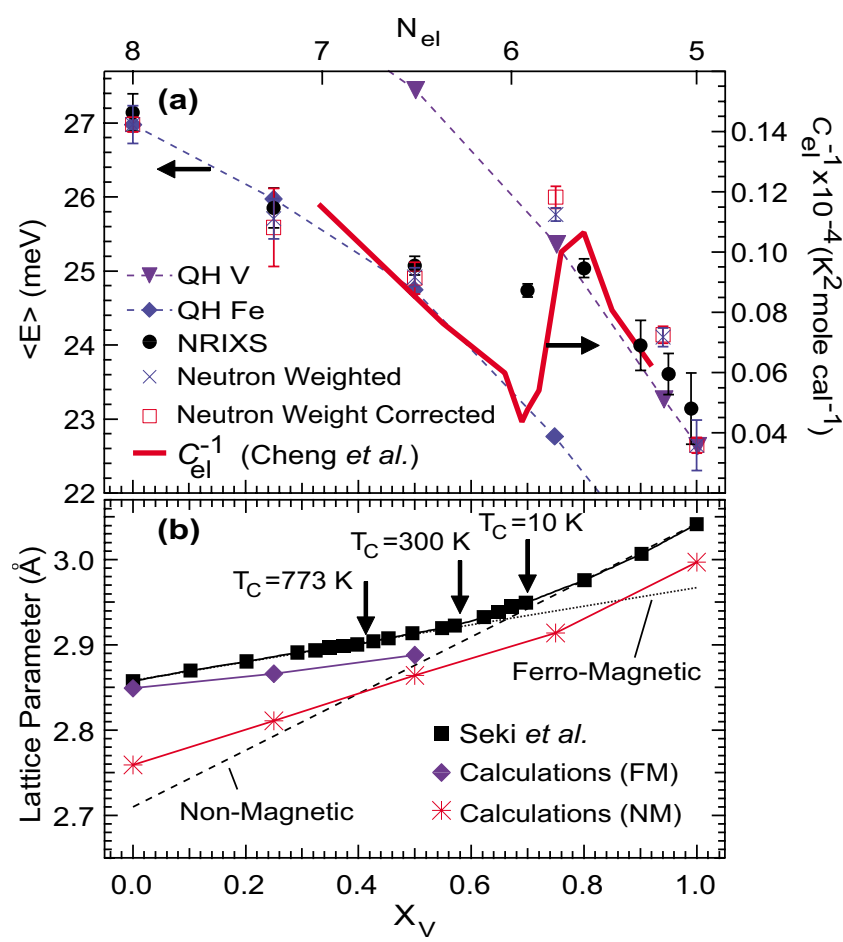

FIG. 5. (Color online) (a) Left axis: average phonon energy from inelastic scattering (markers with an " $\mathrm{X}$ " for neutron-weighted and open squares for neutron-weight corrected) and from NRIXS for samples measured at $300 \mathrm{~K}$ (solid markers with error bars). Dashed lines with markers were obtained from the quasiharmonic predictions using Eq. (3) starting from the volume of pure $\mathrm{Fe}$ (diamonds) and pure $\mathrm{V}$ (triangles). The QH Fe curve reaches $20.3 \mathrm{meV}$ at pure $\mathrm{V}$ and the QH V curve reaches 30.2 at pure Fe. Right axis: inverse of the electronic contribution to the low-temperature heat capacity (Ref. 31) shown as a thick solid line. (b) Lattice parameter of Fe-V alloys from Seki et al. (Ref. 8) at $300 \mathrm{~K}$ and from our DFT calculations. Dashed lines are extrapolations of the experimental results from Seki (Ref. 8) on Fe-rich (ferromagnetic) and V-rich (nonmagnetic) alloys. Arrows show the compositions of the Curie temperature at 10, 300, and $773 \mathrm{~K}$ [from Andersson (Ref. 13)].

$$
\gamma=\frac{3 \alpha V B_{S}}{C_{P}}
$$

with $\alpha$ the linear coefficient of thermal expansion, $V$ the specific volume, $B_{S}$ the isentropic bulk modulus, and $C_{P}$ the constant-pressure heat capacity. ${ }^{32}$

The Grüneisen parameters, $\gamma$, for the alloys at room temperature and $773 \mathrm{~K}$ were determined as follows. The bulk modulus was assessed at room temperature from ultrasonic pulse-echo measurements of longitudinal and transverse wave speeds. The lattice parameter as a function of temperature was measured by x-ray diffractometry. These results, and the derived coefficient of thermal expansion, are listed in Table I. The Grüneisen parameters were obtained with Eq. (4), using the room-temperature heat capacity data compiled by $\mathrm{Smith}^{33}$ and the values in Table I. These results are consistent with prior assessments of Grüneisen parameters for the elements at room temperature; $\gamma=1.66$ for pure Fe (Ref. 34) and $\gamma=1.23$ for pure V. ${ }^{21}$
To account for the quasiharmonic predictions of the phonon DOS curves at $773 \mathrm{~K}$, the $300 \mathrm{~K}$ DOS curves of Fig. 3 were rescaled in energy using Eq. (3) with the values of $\gamma$ and the lattice parameters listed in Table I. Figure 3 shows that the quasiharmonic model only partly accounts for the thermal phonon softening in Fe between 300 and $773 \mathrm{~K}$ but overestimates the phonon softening for $\mathrm{V}$ and $\mathrm{Fe}_{0.06} \mathrm{~V}_{0.94}$. The phonon DOS curves for $\mathrm{Fe}_{0.75} \mathrm{~V}_{0.25}$ and $\mathrm{Fe}_{0.25} \mathrm{~V}_{0.75}$ at $773 \mathrm{~K}$ match well the quasiharmonic prediction.

When alloying $\mathrm{Fe}$ with $\mathrm{V}$, there is an increase in lattice parameter. In a simple application of Eq. (3), we used the alloy volumes and Grüneisen parameters of Table I to predict the change in $\langle E\rangle$ of pure $\mathrm{Fe}$ for the changes in volume with composition. Also accounted for were changes in mass with composition. The same procedure was followed to predict the change in $\langle E\rangle$ of pure $\mathrm{V}$ with composition. The results, shown in Fig. 5, appear generally successful in the Fe-rich and $\mathrm{V}$-rich regions but are not without problems as discussed below.

\section{FIRST-PRINCIPLES SIMULATIONS}

Electronic-structure calculations on ordered structures of Fe-V (Ref. 35) were performed with density-functional theory (DFT) in the generalized gradient approximation. ${ }^{36}$ For all calculations we used ultrasoft pseudopotentials, ${ }^{37}$ treating semicore $3 s$ and $3 p$ orbitals as valence states. An energy cutoff of 30 Ry was used for the plane-wave expansion. The calculations were performed on relaxed structures, where the total energy versus volume was fit to an equation of state to determine the equilibrium volume and bulk modulus. For B2 cubic FeV we obtained $a_{0}=2.891 \AA$, which underestimates the experimental value ${ }^{38}$ by less than $1 \%$. Calculated lattice parameters of bcc $\mathrm{Fe}$ and $\mathrm{V}$ were $a_{0}$ $=2.861 \AA$ and $a_{0}=2.996 \AA$, also in a good agreement with experiment. The ferromagnetic phase was found to minimize the total electronic energy for $\mathrm{Fe}, \mathrm{Fe}_{3} \mathrm{~V}$, and $\mathrm{FeV}$, consistent with experimental magnetic properties ${ }^{13}$ and the calculations of Moruzzi and Marcus for Fe. ${ }^{16}$ Electronic properties were computed using Marzari-Vanderbilt ${ }^{39}$ cold smearing of 0.005 Ry and Monkhorst-Pack ${ }^{40} k$-point grids of $28 \times 28 \times 28,10$ $\times 20 \times 20,22 \times 22 \times 22,10 \times 20 \times 20$, and $28 \times 28 \times 28$ for bcc $\mathrm{Fe}, \mathrm{DO}_{3} \mathrm{Fe}_{3} \mathrm{~V}, \mathrm{~B} 2$ cubic $\mathrm{FeV}, \mathrm{DO}_{3} \mathrm{FeV}_{3}$, and bcc V, respectively. Results are presented in Table II and graphed in Fig. 6.

Phonon frequencies and eigenmodes were calculated using the linear response technique. ${ }^{41}$ For integration in the Brillouin zone, Monkhorst-Pack ${ }^{40}$ grids and Marzari-Vanderbilt ${ }^{39}$ smearing of 0.015 Ry were selected so the error in phonon frequencies would not exceed $1 \%$. For bcc Fe, $\mathrm{DO}_{3} \mathrm{Fe}_{3} \mathrm{~V}, \mathrm{~B} 2$ cubic $\mathrm{FeV}, \mathrm{DO}_{3} \mathrm{FeV}_{3}$, and bcc V, we chose $20 \times 20 \times 20,8 \times 16 \times 16,16 \times 16 \times 16,8 \times 16 \times 16$, and $24 \times 24 \times 24 k$-point sampling, respectively. The dynamical matrices for calculations on fine meshes were obtained from a Fourier interpolation of the dynamical matrices computed on $4 \times 4 \times 4$ phonon meshes. Phonon densities of states then were computed on $28 \times 28 \times 28$ meshes using the tetrahedron scheme.

The results of the phonon calculations are shown in Fig. 7 along with diagrams of the ordered structures. The average 
TABLE I. Temperature and composition dependence of the lattice parameter $( \pm 0.003 \AA)$, coefficient of linear thermal expansion $\left( \pm 0.5 \times 10^{6} \mathrm{~K}^{-1}\right)$, bulk modulus $( \pm 5 \mathrm{GPa})$, and Grüneisen parameters $( \pm 0.09)$ for $\mathrm{Fe}_{1-x} \mathrm{~V}_{x}$ alloys.

\begin{tabular}{lccccccc}
\hline \hline & & \multicolumn{7}{c}{$x$} \\
\cline { 3 - 8 } & $(\mathrm{K})$ & 0 & 0.25 & 0.50 & 0.75 & 0.94 & 1 \\
\hline$a(\AA)$ & 300 & 2.858 & 2.886 & 2.914 & 2.963 & 3.020 & 3.042 \\
& 773 & 2.880 & 2.906 & & 2.980 & 3.036 & 3.057 \\
$\alpha\left(\times 10^{6} \mathrm{~K}^{-1}\right)$ & 300 & 11.8 & 11.0 & 10.1 & 9.3 & 8.6 & 8.4 \\
& 773 & 16.2 & 14.9 & & 12.2 & 11.2 & 10.9 \\
$B_{S}(\mathrm{GPa})$ & 300 & 170 & 166 & 161 & 182 & 172 & 163 \\
$\gamma$ & 300 & 1.71 & 1.58 & 1.45 & 1.58 & 1.42 & 1.23 \\
& 773 & 2.39 & 2.19 & & 2.12 & 1.88 & 1.62 \\
\hline \hline
\end{tabular}

phonon energies are given in Table II. Proper weighting of the calculated pDOS using Eq. (2) and convolution with the experimental resolution function gives the compositional trends found for the measured spectra (Fig. 8), even though the calculations were on ordered structures and the measurements were on solid solutions. For the NRIXS spectra the calculated and measured shapes are similar but the calculations show an Fe pDOS shifted to lower energies than the $\mathrm{V}$ pDOS, whereas the experimental results do not show a difference between the average energy of the Fe pDOS and the total DOS, implying a small shift at most. The calculations showed that the pDOS curves were sensitive to the local chemical environment. For example, the $\mathrm{DO}_{3}$ ordered structure for $\mathrm{Fe}_{3} \mathrm{~V}$ has two different chemical environments for the $\mathrm{Fe}$ atom, and $\mathrm{Fe}$ atoms in these two environments had different pDOS curves and values of $\langle E\rangle$.

\section{DISCUSSION}

\section{A. V-rich alloys}

The phonon DOS curves of Figs. 3 and 4 show a large phonon stiffening when $\mathrm{Fe}$ is added to pure $\mathrm{V}$, at least from pure $\mathrm{V}$ to the composition $\mathrm{Fe}_{0.25} \mathrm{~V}_{0.75}$. From the right side of Fig. 5, we see that the stiffening from adding $\mathrm{Fe}$ is consistent with the quasiharmonic prediction from Eq. (3). However, the $\langle E\rangle$ of the neutron measurements are larger than those predicted by volume effects alone. From prior work on V alloys, ${ }^{20,21,42,43}$ we know that this excess phonon stiffening with small $\mathrm{Fe}$ concentrations originates from a reduction in the electron-phonon coupling..$^{20,21}$

The two DOS curves for pure $\mathrm{V}$ and for $\mathrm{Fe}_{0.06} \mathrm{~V}_{0.94}$ between 300 and $773 \mathrm{~K}$, shown in Fig. 3, do not follow the quasiharmonic softening expected from thermal expansion.

TABLE II. Results of spin-polarized electronic-structure calculations on ordered Fe-V alloys. Results from nonspin-polarized calculations are given in parentheses. The integrated number of electrons for the total eDOS $N$, spin up $N_{+}$, and spin down $N_{-}$, are in units of $e^{-} /$atom. The corresponding bcc lattice parameter is $a_{\mathrm{bcc}}$. The charge transfer $\Delta N$ is $N-N^{\text {element }}$. The total number of electrons at the Fermi level $n\left(E_{\mathrm{F}}\right)$ is in units of states/atom/eV, as is the number of $s$ electrons at the Fermi level $n_{s}\left(E_{\mathrm{F}}\right)$. The first moment of the DOS $\langle E\rangle$ from the phonon calculations are given in $\mathrm{meV}$.

\begin{tabular}{|c|c|c|c|c|c|c|c|c|c|c|c|}
\hline Formula & Atom & Site & $\begin{array}{l}a_{\mathrm{bcc}} \\
(\AA)\end{array}$ & $N$ & $\Delta N$ & $N_{+}$ & $N_{-}$ & $\mu_{B}$ & $n\left(E_{\mathrm{F}}\right)$ & $n_{s}\left(E_{\mathrm{F}}\right)$ & $\langle E\rangle$ \\
\hline $\mathrm{Fe}$ & $\mathrm{Fe}$ & $\mathrm{Fe}$ & $2.849(2.759)$ & 8.00 & & 5.22 & 2.76 & 2.45 & $1.15(3.23)$ & $0.011(0.003)$ & 26.7 \\
\hline $\mathrm{Fe}_{3} \mathrm{~V}$ & Total & & $2.866(2.811)$ & 7.25 & & 4.26 & 2.99 & 1.27 & 0.65 (3.79) & $0.019(0.014)$ & 27.8 \\
\hline $\mathrm{Fe}_{3} \mathrm{~V}$ & $\mathrm{Fe}$ & $4 \mathrm{~V} 1 \mathrm{nn}$ & & 8.09 (8.09) & $+0.09(+0.09)$ & 5.03 & 3.06 & 1.97 & $0.69(3.43)$ & $0.035(0.011)$ & 27.4 \\
\hline $\mathrm{Fe}_{3} \mathrm{~V}$ & $\mathrm{Fe}$ & $8 \mathrm{Fe} 1 \mathrm{nn}$ & & $8.01(8.10)$ & $+0.02(+0.10)$ & 5.28 & 2.73 & 2.55 & $0.47(6.72)$ & $0.003(0.022)$ & 28.9 \\
\hline $\mathrm{Fe}_{3} \mathrm{~V}$ & V & $8 \mathrm{Fe} 1 \mathrm{nn}$ & & $4.80(4.75)$ & $-0.20(-0.25)$ & 1.71 & 3.09 & -1.39 & $0.77(1.58)$ & $0.001(0.018)$ & 27.7 \\
\hline $\mathrm{FeV}$ & Total & & $2.888(2.864)$ & 6.50 & & 3.45 & 3.05 & 0.40 & $1.11(2.83)$ & $0.017(0.038)$ & 23.8 \\
\hline $\mathrm{FeV}$ & $\mathrm{Fe}$ & $\mathrm{Fe}$ & & $8.17(8.20)$ & $+0.17(+0.20)$ & 4.83 & 3.34 & 1.50 & $0.94(3.16)$ & $0.025(0.053)$ & 20.9 \\
\hline $\mathrm{FeV}$ & V & V & & $4.83(4.80)$ & $-0.17(-0.20)$ & 2.07 & 2.76 & -0.69 & $1.28(2.50)$ & $0.008(0.012)$ & 26.7 \\
\hline $\mathrm{FeV}_{3}$ & Total & & 2.914 & 5.75 & & 2.88 & 2.88 & 0.00 & 0.66 & 0.007 & 28.9 \\
\hline $\mathrm{FeV}_{3}$ & $\mathrm{~V}$ & $4 \mathrm{Fe} 1 \mathrm{nn}$ & & 4.88 & -0.20 & 2.44 & 2.44 & 0.00 & 0.46 & 0.007 & 28.6 \\
\hline $\mathrm{FeV}_{3}$ & $\mathrm{~V}$ & $8 \mathrm{~V}$ 1nn & & 4.97 & -0.03 & 2.49 & 2.49 & 0.00 & 0.94 & 0.010 & 31.1 \\
\hline $\mathrm{FeV}_{3}$ & $\mathrm{Fe}$ & $8 \mathrm{~V} 1 \mathrm{nn}$ & & 8.27 & +0.27 & 4.14 & 4.14 & 0.00 & 0.78 & 0.004 & 27.2 \\
\hline V & $\mathrm{V}$ & $\mathrm{V}$ & 2.997 & 5.00 & & 2.50 & 2.50 & 0.00 & 1.95 & 0.018 & 21.8 \\
\hline
\end{tabular}




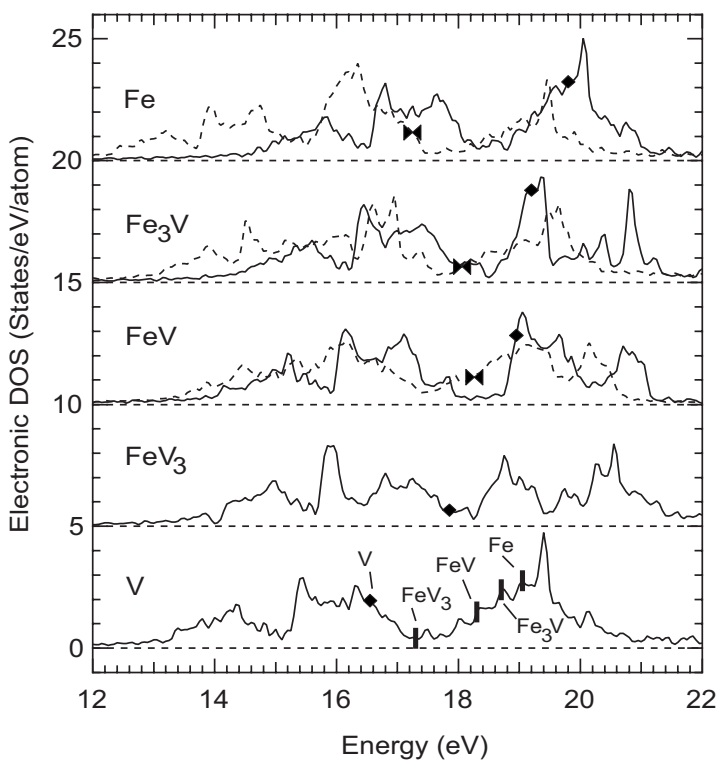

FIG. 6. Calculated eDOS for Fe, V, and ordered Fe-V alloys. Nonmagnetic are solid curves with the Fermi level at the diamonds. Magnetic results are dashed curves with the Fermi level at bowties. The curve for $\mathrm{V}$ shows the Fermi levels of $\mathrm{V}$ and for the other alloys in the rigid band approximation using the total number of electrons, 5, 5.75, 6.5, 7.25, and 8 for $\mathrm{V}, \mathrm{FeV}_{3}, \mathrm{FeV}$, and $\mathrm{Fe}_{3} \mathrm{~V}$, and $\mathrm{Fe}$, respectively. Curves are offset by integer multiples of 5 states/eV/atom.

This is the result of a strong electron-phonon coupling in $\mathrm{V}$ at low temperatures. Specifically, at low temperatures the adiabatic electron-phonon coupling causes a softening of the phonons, and with increasing temperature the electronphonon coupling becomes weaker, causing a stiffening effect that counteracts the quasiharmonic softening. Upon alloying with $\mathrm{Fe}$, there is a depletion of the number of states at the Fermi level, $n\left(E_{\mathrm{F}}\right)$, reducing the number of electronic states available for screening the displacements of atoms. With the loss of this screening of charge disturbances caused by nuclear displacements (an electron-phonon interaction), there is a greater sensitivity of the electronic energy to atom displacements, and stiffer interatomic forces. One trend supporting this explanation is the increase in the inverse electronic heat capacity with $\mathrm{Fe}$ concentration, $C_{\mathrm{el}}^{-1}$, shown in Fig. 5. Also, Fig. 6 shows a reduction in $n\left(E_{\mathrm{F}}\right)$ from the addition of $\mathrm{Fe}$ to $\mathrm{V}$, by comparing calculations on pure $\mathrm{V}$ with the compound $\mathrm{V}_{3} \mathrm{Fe}$, or with the simpler rigid band filling of the eDOS of pure $\mathrm{V}$ from the extra electrons of $\mathrm{Fe}$. The rigid band model also does reasonably well at giving $n\left(E_{\mathrm{F}}\right)$ for the nonmagnetic calculations of all compounds, predicting a reduction of $n\left(E_{\mathrm{F}}\right)$ from $\mathrm{V}$ to $\mathrm{FeV}_{3}$, followed by an increase from $\mathrm{FeV}_{3}$ to pure $\mathrm{Fe}$. This trend is modified significantly by the occurrence of ferromagnetism at higher Fe concentrations, as discussed below.

For lower concentrations of $\mathrm{Fe}$ in bcc V, both the calculations and the experimental results show a bunching of phonon energies around $25 \mathrm{meV}$ for the $\mathrm{Fe}$ pDOS. This might look reminiscent of a resonance mode of a heavy impurity in a light matrix, but the masses of $\mathrm{Fe}$ and $\mathrm{V}$ are too similar for such an explanation. The resonance mode is also promoted

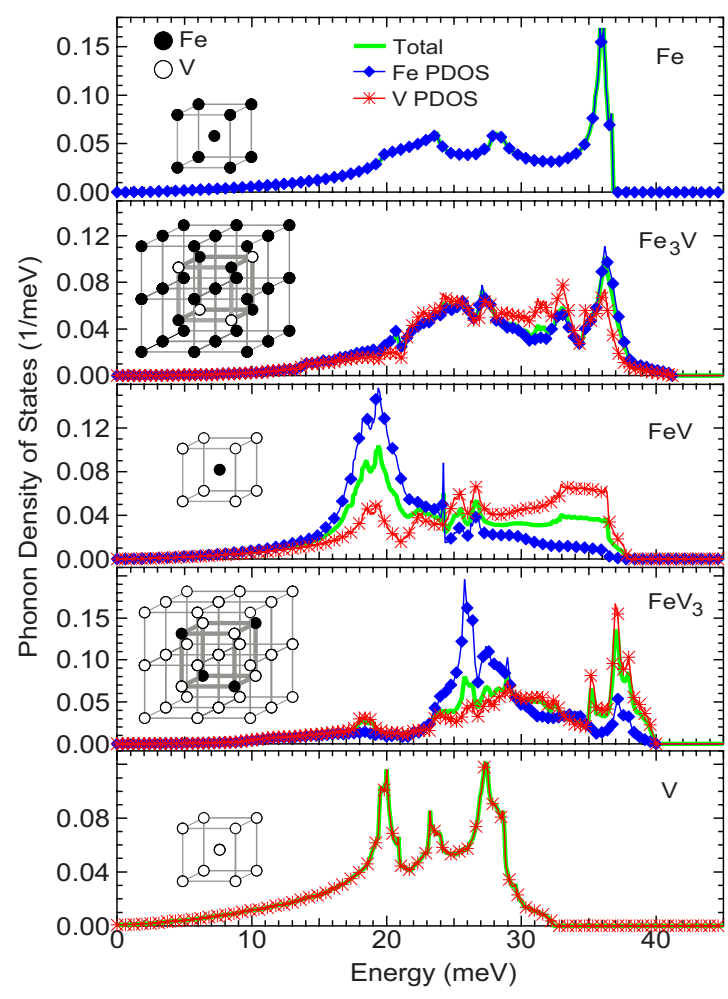

FIG. 7. (Color online) Calculated phonon DOS for ordered bccbased structures. Inset is the structure of $\mathrm{Fe}$ atoms (black) and $\mathrm{V}$ atoms (white). The total DOS (thick solid curve) is the concentration-weighted sum of the partial DOS of Fe (diamonds) and $\mathrm{V}$ (stars).

by weaker interatomic forces between impurity and host, which typically lower the frequency of the mode. This does not seem to be the case for Fe in V, since the average energy of the Fe pDOS is the same as the average energy of the total phonon DOS. The calculations on the ordered structures do predict a difference in the average energies of the $\mathrm{Fe}$ and $\mathrm{V}$ pDOS, especially for $\mathrm{FeV}_{3}$ and $\mathrm{FeV}$. Although we do not rule

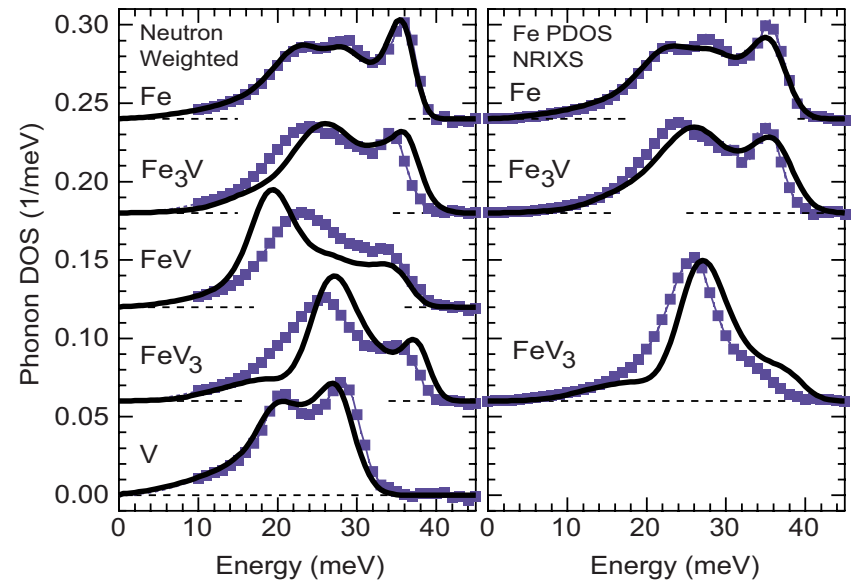

FIG. 8. (Color online) Calculated neutron-weighted phonon DOS (left) and Fe partial phonon DOS (right) for ordered bcc-based structures convoluted with experimental resolution functions (thick solid curves) and compared to measured curves from disordered alloys. 
out the resonance mode explanation, we suggest instead that as the local volume around an $\mathrm{Fe}$ atom becomes larger for V-rich alloys, the local forces on the $\mathrm{Fe}$ atoms become more isotropic and less sensitive to the direction of motion with respect to the neighboring atoms. This should also tend to decouple the phases of Fe polarizations from the matrix $\mathrm{V}$ atoms. A consequence is that the energies for the transverse and longitudinal polarizations of $\mathrm{Fe}$ motions tend to merge together as the local volume for the $\mathrm{Fe}$ atom becomes larger.

\section{B. Phonons in ferromagnetic alloys}

Pure iron is ferromagnetic at room temperature with a magnetic moment of $2.2 \mu_{\mathrm{B}}$ /atom. The Curie temperature decreases with $\mathrm{V}$ concentration and reaches $300 \mathrm{~K}$ near $58 \%$ V. ${ }^{13,44,45}$ Increasing the vanadium concentration also causes a linear decrease in the average alloy moment approximately as expected for dilution with nonmagnetic atoms although $\mathrm{V}$ has a small moment that aligns antiparallel to the $\mathrm{Fe}$ moments. ${ }^{10,17,19,46,47}$ Our calculations are consistent with these reports and predict that the lattice parameter of the magnetic phase is larger than that of the nonmagnetic phase for most compositions [Fig. 5(b)]. The effect of magnetism on the phonon DOS may generally include a contribution from the change in volume, where Eq. (3) predicts that the phonons would be softer in the ferromagnetic alloys. The total energy versus volume calculations of Moruzzi and Marcus ${ }^{16}$ predicted a lower volume and higher bulk modulus for nonspin-polarized Fe. This is consistent with our $\mathrm{QH}$ prediction in Fig. 5(a), which shows the extrapolated behavior of the nonmagnetic alloys to have higher average energies than the ferromagnetic alloys. However, the calculations and extrapolations of Fig. 5(b) indicate a crossover between the specific volumes for the magnetic and nonmagnetic phases at approximately the onset of ferromagnetism so this volume effect may be smaller for intermediate compositions.

We note from Fig. 3 that the low transverse acoustic modes in the energy range $10-23 \mathrm{meV}$ tend to soften relatively rapidly with temperature in $\mathrm{Fe}$ and $\mathrm{Fe}_{0.75} \mathrm{~V}_{0.25}$, indicative of an anharmonic effect. This is consistent with the neutron-scattering measurements of Satija et $a l .{ }^{48}$ and the elastic constant measurements of Dever, ${ }^{49}$ which show a kink in the softening at the Curie temperature. The excess softening of the low transverse modes is also captured in the calculation by Hasegawa et al. ${ }^{50}$ which the authors attributed to a softening of the second-nearest-neighbor force constant. Although the low transverse modes soften quickly with temperature, other modes may exhibit typical quasiharmonic softening. A more complete study of the temperature dependence of thermal phonon softening in Fe would be useful.

\section{Composition dependence of phonons across the ferromagnetic transition}

A large change in the electronic specific heat, as shown by the discontinuity in $C_{\mathrm{el}}^{-1}$ in Fig. 5, is found near the magnetic transition (at cryogenic temperatures this occurs at approximately $75 \% \mathrm{~V})$. This increase in $C_{\mathrm{el}}$ with ferromagnetism indicates an increase in $n\left(E_{\mathrm{F}}\right),{ }^{51,52}$ and correlates well to the phonon softening. However, our ab initio calculations show a large decrease calculated for $n\left(E_{\mathrm{F}}\right)$ with ferromagnetism (Fig. 6), the opposite trend. Evidently our calculations for $n\left(E_{\mathrm{F}}\right)$ with alloy composition are not reliable across the ferromagnetic transition. One reason is that the calculations were performed on ordered compounds, whereas the samples were largely disordered chemically. Another concern is that the calculations were performed for nonmagnetic states without moments at the Fe atoms, and were not states with disordered moment orientations. Finally, the screening characteristics of the electrons near the Fermi surface vary with $\vec{k}$, and more detail may be required than $n\left(E_{\mathrm{F}}\right)$ alone. However, since the phonon softening did correlate well with the discontinuity in $C_{\mathrm{el}}^{-1}$ in Fig. 5, we attribute much of the discrepancy of the $a b$ initio calculations to an improper treatment of chemical and magnetic disorder in the materials.

At low temperatures the enthalpic term dominates the Gibbs free energy $(G=H-T S$, where $H$ is the enthalpy, $T$ the temperature, and $S$ the entropy). The energy versus volume curves for different magnetic states from first principles provides valuable insight into the low-temperature magnetic phase stability, where for pure Fe the ferromagnetic phase minimizes the energy. However, at moderate temperatures entropic effects begin to dominate. Figure 5 shows that phonons in the ferromagnetic phase have lower average energies than those in the nonmagnetic phase for $\mathrm{Fe}-\mathrm{V}$ alloys. The vibrational entropy is larger for softer DOS curves (i.e., when there are more phonon states at lower energies). ${ }^{1}$ At moderate temperatures the ferromagnetic phase is stabilized with respect to the nonmagnetic phase by the vibrational entropy, which from the trend lines of Fig. 5(a) is approximately $0.3 k_{\mathrm{B}}$ /atom. In contrast, the electronic entropy destabilizes the ferromagnetic phase by $0.1-0.2 k_{\mathrm{B}} /$ atom, as the number of states at the Fermi level is greatly reduced by spin polarization.

\section{CONCLUSIONS}

Time-of-flight INS measurements were performed at 300 and $773 \mathrm{~K}$. The synchrotron Mössbauer technique, NRIXS, was used to probe the Fe pDOS at room temperature. Interpretations of the compositional trends were supported by $a b$ initio lattice dynamics calculations on ordered structures of similar compositions. The quasiharmonic model gave good overall agreement for the compositional trends of the phonon stiffness for V-rich and Fe-rich alloys. The change in the phonon stiffness across the ferromagnetic transition was interpreted with partial success to be a result of changes in the electronic density of states (eDOS) at the Fermi level, $n\left(E_{\mathrm{F}}\right)$, due to the onset of ferromagnetism. This interpretation was supported by $a b$ initio calculations and from prior results on the electronic specific heat. In general, the greater $n\left(E_{\mathrm{F}}\right)$, the more effective the electronic screening and the softer the phonons. The quasiharmonic model could account for the temperature dependence of the phonons at intermediate alloy compositions but large departures at the V-rich compositions were observed owing to adiabatic electron-phonon coupling and at the Fe-rich compositions owing to other anharmonic effects. At more dilute Fe concentrations, the Fe pDOS tended toward a single peak centered around $25 \mathrm{meV}$, which 
we suggest is indicative of more isotropic dynamics of $\mathrm{Fe}$ atoms when in larger atomic volumes.

\section{ACKNOWLEDGMENTS}

The portions of this work conducted at Oak Ridge National Laboratory were supported by the Scientific User Facilities Division and by the Division of Materials Sciences and Engineering, Office of Basic Energy Sciences, DOE. This work was supported by the Department of Energy through the Basic Energy Sciences under Grant No. DEFG02-03ER46055 and BES-MS under Grant No. W-31-109ENG-38. Portions of this work were performed at HPCAT (Sector 16), Advanced Photon Source (APS), Argonne National Laboratory. HPCAT is supported by CIW, CDAC, UNLV, and LLNL through funding from DOE-NNSA, DOEBES, and NSF. Use of the APS was supported by DOE-BES under Contract No. DE-AC02-06CH11357. This work benefited from DANSE software developed under NSF under Grant No. DMR-0520547.
${ }^{1}$ B. Fultz, Prog. Mater. Sci. 55, 247 (2010).

${ }^{2}$ O. Delaire, M. S. Lucas, A. M. dos Santos, A. Subedi, A. S. Sefat, M. A. McGuire, L. Mauger, J. A. Muñoz, C. A. Tulk, Y. Xiao, M. Somayazulu, J. Y. Zhao, W. Sturhahn, E. E. Alp, D. J. Singh, B. C. Sales, D. Mandrus, and T. Egami, Phys. Rev. B 81, 094504 (2010).

${ }^{3}$ M. S. Lucas, O. Delaire, M. L. Winterrose, T. Swan-Wood, M. Kresch, I. Halevy, B. Fultz, J. Hu, M. Lerche, M. Y. Hu, and M. Somayazulu, Phys. Rev. B 80, 214303 (2009).

${ }^{4}$ M. Kresch, M. Lucas, O. Delaire, J. Y. Y. Lin, and B. Fultz, Phys. Rev. B 77, 024301 (2008).

${ }^{5}$ M. Kresch, O. Delaire, R. Stevens, J. Y. Y. Lin, and B. Fultz, Phys. Rev. B 75, 104301 (2007).

${ }^{6}$ O. Delaire, A. F. May, M. A. McGuire, W. D. Porter, M. S. Lucas, M. B. Stone, D. L. Abernathy, V. A. Ravi, S. A. Firdosy, and G. J. Snyder, Phys. Rev. B 80, 184302 (2009).

${ }^{7}$ H. K. Mao, J. Xu, V. V. Struzhkin, J. Shu, R. J. Hemley, W. Sturhahn, M. Y. Hu, E. E. Alp, L. Vocadlo, D. Alfè, G. D. Price, M. J. Gillan, M. Schwoerer-Böhning, D. Häusermann, P. Eng, G. Shen, H. Giefers, R. Lübbers, and G. Wortmann, Science 292 , 914 (2001).

${ }^{8}$ J. I. Seki, M. Hagiwara, and T. Suzuki, J. Mater. Sci. 14, 2404 (1979).

${ }^{9}$ J. M. Sanchez, M. C. Cadeville, V. Pierron-Bohnes, and G. Inden, Phys. Rev. B 54, 8958 (1996).

${ }^{10}$ M. V. Nevitt and A. T. Aldred, J. Appl. Phys. 34, 463 (1963).

${ }^{11}$ R. M. Bozorth, Ferromagnetism (Van Nostrand, New York, 1951), p. 258.

${ }^{12}$ J. C. Krause, J. Schaf, M. I. da Costa, and C. Paduani, Phys. Rev. B 61, 6196 (2000).

${ }^{13}$ J.-O. Andersson, CALPHAD: Comput. Coupling Phase Diagrams Thermochem. 7, 305 (1983).

${ }^{14}$ A. Mustaffa and D. A. Reed, J. Magn. Magn. Mater. 5, 349 (1977).

${ }^{15}$ R. G. Jordan, X. Wang, A. M. Begley, S. L. Qiu, and Y. Liu, Solid State Commun. 78, 1045 (1991).

${ }^{16}$ V. L. Moruzzi and P. M. Marcus, Phys. Rev. B 45, 2934 (1992).

${ }^{17}$ D. D. Johnson, F. J. Pinski, and J. B. Staunton, J. Appl. Phys. 61, 3715 (1987).

${ }^{18}$ F. Kajzar, J. Phys. F: Met. Phys. 7, 1623 (1977).

${ }^{19}$ I. Mirebeau and G. Parette, J. Appl. Phys. 53, 1960 (1982).

${ }^{20}$ O. Delaire, M. S. Lucas, J. A. Muñoz, M. Kresch, and B. Fultz, Phys. Rev. Lett. 101, 105504 (2008).

${ }^{21}$ O. Delaire, M. Kresch, J. A. Muñoz, M. S. Lucas, J. Y. Y. Lin, and B. Fultz, Phys. Rev. B 77, 214112 (2008).
${ }^{22}$ E. E. Alp, W. Sturhahn, T. S. Toellner, J. Zhao, M. Hu, and D. E. Brown, Hyperfine Interact. 144, 3 (2002).

${ }^{23}$ M. Seto, Y. Yoda, S. Kikuta, X. W. Zhang, and M. Ando, Phys. Rev. Lett. 74, 3828 (1995).

${ }^{24}$ W. Sturhahn, T. S. Toellner, E. E. Alp, X. Zhang, M. Ando, Y. Yoda, S. Kikuta, M. Seto, C. W. Kimball, and B. Dabrowski, Phys. Rev. Lett. 74, 3832 (1995).

${ }^{25}$ W. Sturhahn, Hyperfine Interact. 125, 149 (2000).

${ }^{26}$ V. F. Sears, E. C. Svensson, and B. M. Powell, Can. J. Phys. 73, 726 (1995).

${ }^{27}$ M. G. Kresch, Ph.D. thesis, California Institute of Technology, 2009.

${ }^{28}$ B. Fultz, T. Kelley, J. Lin, J. Lee, O. Delaire, M. Kresch, M. McKerns, and M. Aivazis, "Experimental inelastic neutron scattering: Introduction to DANSE," http://drchops.caltech.edu/Docs (2009).

${ }^{29}$ R. E. Hanneman and A. N. Mariano, Trans. Metall. Soc. AIME 230, 937 (1964).

${ }^{30}$ T. V. Philip and P. Beck, Trans. Metall. Soc. AIME 209, 1269 (1957).

${ }^{31}$ C. H. Cheng, C. T. Wei, and P. A. Beck, Phys. Rev. 120, 426 (1960).

${ }^{32}$ G. Grimvall, Thermophysical Properties of Materials, rev. ed. (North-Holland, Amsterdam, 1999).

${ }^{33}$ J. F. Smith, Bull. Alloys Phase Diagrams 5, 184 (1984).

${ }^{34}$ R. Boehler and J. Ramakrishnan, J. Geophys. Res. 85, 6996 (1980).

${ }^{35}$ P. Giannozzi, S. Baroni, N. Bonini, M. Calandra, R. Car, C. Cavazzoni, D. Ceresoli, G. L. Chiarotti, M. Cococcioni, I. Dabo, A. Dal Corso, S. de Gironcoli, S. Fabris, G. Fratesi, R. Gebauer, U. Gerstmann, C. Gougoussis, A. Kokali, M. Lazzeri, L. MartinSamos, N. Marzari, F. Mauri, R. Mazzarello, S. Paolini, A. Pasquarello, L. Paulatto, C. Sbraccia, S. Scandolo, G. Sclauzero, A. P. Seitsonen, A. Smogunov, P. Umari, and R. M. Wentzcovitch, J. Phys.: Condens. Matter 21, 395502 (2009).

${ }^{36}$ J. P. Perdew, K. Burke, and M. Ernzerhof, Phys. Rev. Lett. 77, 3865 (1996).

${ }^{37}$ D. Vanderbilt, Phys. Rev. B 41, 7892 (1990).

${ }^{38}$ P. E. A. Turchi, L. Reinhard, and G. M. Stocks, Phys. Rev. B 50, 15542 (1994).

${ }^{39}$ N. Marzari, D. Vanderbilt, A. De Vita, and M. C. Payne, Phys. Rev. Lett. 82, 3296 (1999).

${ }^{40}$ H. J. Monkhorst and J. D. Pack, Phys. Rev. B 13, 5188 (1976).

${ }^{41}$ P. Giannozzi, S. de Gironcoli, P. Pavone, and S. Baroni, Phys. Rev. B 43, 7231 (1991). 
${ }^{42}$ O. Delaire and B. Fultz, Phys. Rev. Lett. 97, 245701 (2006).

${ }^{43}$ P. D. Bogdanoff, B. Fultz, J. L. Robertson, and L. Crow, Phys. Rev. B 65, 014303 (2001).

${ }^{44}$ S. Arajs, R. V. Colvin, H. Chessin, and J. M. Peck, J. Appl. Phys. 33, 1353 (1962).

${ }^{45}$ Y. Kakehashi, J. Magn. Magn. Mater. 54-57, 1096 (1986).

${ }^{46}$ M. Fallot, Ann. Phys. 6, 305 (1936).

${ }^{47}$ A. M. van der Kraan, D. B. de Mooij, and K. H. J. Buschow,
Phys. Status Solidi A 88, 231 (1985).

${ }^{48}$ S. K. Satija, R. P. Comés, and G. Shirane, Phys. Rev. B 32, 3309 (1985).

${ }^{49}$ D. J. Dever, J. Appl. Phys. 43, 3293 (1972).

${ }^{50}$ H. Hasegawa, M. W. Finnis, and D. G. Pettifor, J. Phys. F: Met. Phys. 17, 2049 (1987).

${ }^{51}$ M. F. Manning, Phys. Rev. 63, 190 (1943).

${ }^{52}$ E. C. Stoner, Proc. R. Soc. London 154, 656 (1936). 